\title{
Dobrow syndrome
}

INSERM

\section{Source}

INSERM. (1999). Orphanet: an online rare disease and orphan drug data base. Dobrow syndrome. ORPHA:3262

Dobrow syndrome is a rare multiple congenital defects/dysmorphic syndrome characterized by variable degrees of bony syng nathia associated with variable additional abnormalities, including growth retardation, intellectual disability, microcephaly, iris coloboma, nystagmus, deafness, and vertebral segmentation defects, as well as genital, limb and additional facial malformations, among others. 